\title{
A need for Recalibration of the Discussions on Access and
}

\section{Benefit Sharing}

Rodrigo Sara ${ }^{1, \#}$, Markus Wyss ${ }^{2, \#, *}$, René Custers ${ }^{3}$, Anouk in 't Veld ${ }^{4}$ and Dominic Muyldermans ${ }^{5}$

*Corresponding author: markus.wyss@dsm.com

"These authors contributed equally to this work

\section{Affiliations:}

1. One Planet Solutions, Montpellier, France; 0000-0002-6341-6981

2. DSM Nutritional Products Ltd., Kaiseraugst, Switzerland; 0000-0003-4533-3274

3. VIB, Ghent, Belgium; 0000-0003-1382-3543

4. Robeco Institutional Asset Management, Rotterdam, the Netherlands; 0000-0003-0828-7424

5. Dominic Muyldermans BV, Oostkamp, Belgium; 0000-0002-0518-7359

Conflict of interest statement: The views and opinions expressed in this article are those of the authors in their personal capacity. None of the observations, opinions or conclusions expressed herein are to be attributed to or construed as the views or positions of any organization, association or company. 


\begin{abstract}
Facing unprecedented global declines in the extent and integrity of ecosystems, the $15^{\text {th }}$ UN Biodiversity Conference (COP-15) in Kunming, China, presents an opportunity for transformative change. However, a lack of consensus on two key issues - resource mobilization and Access and Benefit Sharing (ABS) associated with Digital Sequence Information (DSI) - risks stalling negotiations for an ambitious 'Post-2020 Global Biodiversity Framework' as the next 10-year strategic plan under the Convention on Biological Diversity. We highlight systemic misconceptions concerning the financing of biodiversity and the burden this places on the ABS system. In the context of DSI, we caution that conflating ABS with resource mobilization risks disrupting modern science policy built on open access, with potentially severe ramifications for scientific research and innovation. To resolve these tensions, we call for a recalibration of discussions on ABS in order to maximize the value delivered by biodiversity for all of society, including indigenous peoples and local communities.
\end{abstract}

Keywords: Access and Benefit Sharing (ABS); resource mobilization; Digital Sequence Information (DSI); Post-2020 Global Biodiversity Framework; biodiversity crisis 


\section{Time is pressing for bold action on biodiversity}

High hopes and expectations had been placed on 2020 as the 'Super Year for Nature', with the $15^{\text {th }}$ UN Biodiversity Conference (COP-15) in Kunming, China, set to endorse the 'post-2020 global biodiversity framework' (GBF), which will become the next 10-year strategic plan for the Convention on Biological Diversity (CBD). The delays wrought by the SARS-CoV-2 pandemic have not dampened expectations for 2021. Indeed, the ongoing GBF negotiations will likely benefit from the extension of time and the parallel discussions on nature and biodiversity in the context of the UN Decade on Ecosystem Restoration (2021$2030)^{1}$, the $26^{\text {th }}$ UN Climate Change Conference ${ }^{2}$, the UN Food Systems Summit ${ }^{3}$, and the UN Ocean Conference ${ }^{4}$, all taking place this year.

Time is pressing for bold action. We live in times of unprecedented global declines in the extent and integrity of ecosystems and the abundance and number of wild species ${ }^{5}$, which led UN Secretary General António Guterres to proclaim that "humanity is waging war on nature, and we need to rebuild our relationship with it" (UN Summit on Biodiversity, New York, Sept. 30, 2020). According to the President of the UN General Assembly, Volkan Bozkir, "Kunming must do for biodiversity what Paris did for climate change in 2015 , by elevating the discourse to the mainstream and placing it firmly on the political agenda" (UN Summit on Biodiversity, New York, Sept. 30, 2020).

\section{Systemic misconceptions about the financing of biodiversity}

Despite these high hopes, there are major issues that risk stalling negotiations for an ambitious GBF: notably 1) resource mobilization, which refers to the provision of sufficient resources (human, financial, technical, etc.) to achieve the ambitious targets that are needed to afford the intended transformative change, and 2) Access and Benefit Sharing (ABS), referring to the measures under the CBD and its subsidiary agreement, the Nagoya Protocol, to regulate (i) access to genetic resources and associated 
traditional knowledge (ATK) and (ii) the equitable sharing of benefits from their use. In the absence of alignment on these two issues, consensus on the GBF may fail to emerge, thereby putting its adoption, and thus progress on reversing biodiversity loss, at risk.

To realize the high ambitions of the GBF, some assert that up to one trillion USD per year will be required $^{6}$. For the preservation of biodiversity, three different streams of action will be needed: 1 ) conservation of biodiversity through, e.g., protection of species and habitats, and ex situ collections; 2) sustainable use of biodiversity; and 3) restoration of damaged ecosystems. These three streams of action each require a distinct conceptual framework and implementation (including financing) strategy, but they should be complementary.

ABS is first and foremost an integral part of sustainable use of biodiversity and should not be misconstrued as a primary financing mechanism for overall resource mobilization. Yet, failure of the international community so far to set aside the necessary resources for the preservation of biodiversity has contributed to nurturing unrealistic expectations regarding the monetary benefits that should be generated through ABS agreements. By its nature, ABS can and should be expected to contribute only in part to the financial resources needed to achieve the long-term targets of the GBF.

In the ABS realm, parties are also debating whether the scope of access and benefit sharing obligations should be expanded to "Digital Sequence Information" (DSI), an undefined term used as a placeholder in the international discussions that may be interpreted to cover all sequence data and other (digital) information on genetic resources. Those advocating for Open Science and Open Access to published information fear that expansion of the formal scope of benefit sharing obligations to DSI would stifle research and innovation, represent a significant roadblock in addressing global challenges, and, ultimately, undermine the ambitions of the $\mathrm{CBD}$ and $\mathrm{GBF}^{7,8}$. On the other side, providers of genetic resources fear that in an era where DNA sequencing has become so widespread and cheap, not including 
DSI in the formal scope of benefit sharing obligations undermines the principles of prior informed consent under the Nagoya Protocol and the fair sharing of benefits ${ }^{9}$.

We question whether the frustrations surrounding DSI, and ABS more broadly, are in fact proxies for misunderstandings about the value and benefits of genetic resources in the overarching resource mobilization context. Given these tensions, addressing deficiencies in resource mobilization may provide more policy space within ABS discussions, including DSI, thereby relieving polarized positions and enabling potential improvements to $\mathrm{ABS}$ within the $\mathrm{CBD}$ and other international frameworks.

\section{Misplaced burden in the current ABS system}

ABS, which started as an innovative vision of the future of conservation and sustainable use of biological diversity during the negotiations of the $\mathrm{CBD}$, in the late 1980s, has gone beyond its original objective of enabling equity and fairness. The intent of the CBD was to 'facilitate' access to genetic resources and ATK, in order to encourage sustainable and responsible use, with benefits arising from such access and use shared with providers. Today, we are seeing very restrictive regimes that neither facilitate access nor result in substantial sharing of benefits. Implementation experiences during the last decade indicate that ABS obligations have turned out to be a clear bottleneck for collaboration, research and development ${ }^{10-}$

${ }^{13}$. This unintended consequence is a concern for all.

Most users of genetic resources, including biotechnology sectors and the public life sciences research community, have limited impact on biodiversity loss. Very much to the contrary, they often have long-reaching positive impacts on sustainable development, which all parties agree should be enabled and perpetuated. These users are well-placed - and called upon - to alleviate global challenges associated with sustainable development, human and animal health, food security, climate change and biodiversity loss. 
Yet, perversely, these are the same stakeholders that bear the burden of regulatory compliance and high transaction costs associated with the current $\mathrm{ABS}$ regimes under the CBD umbrella. This burden often involves significant human and financial resources in typically lengthy processes for approvals, or, in worst cases, the sheer impossibility to obtain clarity or approvals. The bilateral nature of such regimes, their inherent limitations in generating monetary benefits, the patchwork of different policies and the focus on monetary benefits instead of value creation and sharing mean that the current ABS system is undermining the positive impact of these sectors on sustainable development.

In order to overcome the shortcomings in how ABS is currently conceived and implemented, international policy changes are crucially needed to improve legal certainty, efficiency and workability, thereby providing a solid basis for conservation and sustainable use. In the ongoing policy discussions more multilateral systems have been put forward. This approach is only valid if it is designed to facilitate access while enhancing value-creation and value sharing as mutually reinforcing priorities. This also requires that providers of genetic resources and ATK are empowered to participate in value-creation, to realize bioeconomic opportunities, and to access technologies related thereto, i.a. via targeted capacity building and development.

\section{ABS must be facilitated to enable sustainable use}

Value creation depends on sustainable use of biodiversity, whereby such use cannot be considered truly sustainable without benefit sharing, or more broadly value sharing, in one form or another. Therefore, sustainable use and value sharing are inseparable, and should not be considered as distinct ambitions of the CBD. Sustainable use of the components of biological diversity is defined in Article 2 of the Convention as "the use of components of biological diversity in a way and at a rate that does not lead to the long-term decline of biological diversity, thereby maintaining its potential to meet the needs and 
aspirations of present and future generations". The definition contains two key elements which should be considered in the context of R\&D activities: the first element refers to the use of components of biodiversity which does not lead to a decline in biological diversity. The use of biodiversity, inter alia as genetic resources or DSI in R\&D activities, typically does not deplete biological diversity (on the contrary) and can thus be qualified as sustainable use.

By conducting R\&D, users in the public and private spheres develop innovations that are in line with the second element of sustainable use: use of biodiversity enabling it 'to meet the needs and aspirations of present and future generations'. As exemplified by ongoing societal crises, foundational 'needs for present and future generations' include food security, human health, and a healthy planet. In order to find solutions for these, sustainable use as defined under the CBD relies on effective access and use of genetic resources and the open access and exchange of DSI. Policymakers need to revisit this connection and realize that facilitating ABS, including simplified access measures, directly enables sustainable use, thereby laying the foundation for value creation and value sharing.

\section{We need to recalibrate the resource mobilization and ABS discussions}

We need to learn from the shortcomings of the past. The Aichi Targets were negotiated side-by-side with the Nagoya Protocol as part of a political compromise in 2010. But yet, the implementation of the Strategic Plan for Biodiversity over the previous decade largely failed to meet the Aichi Targets. And the Nagoya Protocol is also a long-way from a win-win. Skeptics could rightfully call for caution when considering another political compromise, this time between the GBF and DSI. Thus, a science-based and measurable GBF is needed which is capable of delivering on the transformative action required to drastically change our relationship with nature. We must get our act straight in taking care of our planet and its biodiversity. 
An effective resource mobilization strategy is crucial for implementation of the GBF, properly addressing and balancing the needs for conservation, sustainable use, and restoration of biodiversity. While ABS should be regarded as an integral part of sustainable use, conservation and restoration require something more apt such as biodiversity impact-based contributions, green investments, or repealing of biodiversity-damaging subsidies, amongst others. These ideas need to be more thoroughly considered and flanked by science-based indicators, which enable an impact assessment and effectively guide the intended transformative change.

Reaching the ambitious goals and targets of the GBF relies upon access to and use of genetic resources, especially the open access and exchange of DSI as the foundation of biodiversity research. Such use of genetic resources and DSI will contribute significant value in the context of these goals and targets. This value sharing includes, inter alia, capacity building, as emphasized by the CBD and the GBF. To enable such sustainable use, we should dare to rethink the current ABS systems that have high transaction costs, fail to generate the desired monetary and non-monetary resources, and have created significant thresholds for sustainable use. The potential for standardization or multilateral benefit sharing schemes should be seriously investigated, including a detailed assessment of their feasibility and effectiveness for all stakeholders. Our criticism is focused on the unintended consequences of (i) restrictive ABS regulatory systems and (ii) potential impediments to open access and exchange of DSI on innovation and research, and not on value sharing as a principle. Such sharing remains important from an ethical and social responsibility point of view.

Resolving tensions with $\mathrm{ABS}$ and resource mobilization also calls for more inclusive participation of all relevant stakeholders to ensure appropriate co-ownership of the challenges that lie ahead, as well as sufficient agency to be part of the solution. This co-ownership must also address mechanisms to share value between countries having highly developed R\&D infrastructures and countries being short of such resources. Governments, indigenous peoples and local communities (IPLCs), users from both the public 
and private sector, as well as the financial sector will need to work in lockstep to ensure that mainstreaming including the integration of negative externalities becomes an integral part of economic models; otherwise, the trillions in investment required to avert the disaster that is being wrought by the convergence of the climate and biodiversity crises will remain out of reach.

The negotiations for the GBF in the lead up to COP-15 provide an opportunity for all stakeholders to work creatively and collaboratively to recalibrate the ABS discussion. However, the clock is ticking and we cannot afford to squander another decade due to a lack of ambition, imagination, a holistic perspective or the ability to compromise. Bold action is required to timely resolve the tensions highlighted in this paper, and to define an ABS approach that is truly fit-for-purpose!

\section{References}

1. https://www.decadeonrestoration.org/

\section{2. https://ukcop26.org/}

\section{3. https://www.un.org/en/food-systems-summit}

\section{4. https://www.un.org/en/conferences/ocean2020}

5. Díaz S. et al. Pervasive human-driven decline of life on Earth points to the need for transformative change. Science 366, eaax3100 (2019).

6. Deutz, A. et al. Financing Nature: Closing the global biodiversity financing gap (The Paulson Institute, The Nature Conservancy, and the Cornell Atkinson Center for Sustainability, 2020).

7. Gaffney, J. et al. Open access to genetic sequence data maximizes value to scientists, farmers, and society. Global Food Security 26, 100411 (2020). 
8. Laird, S. et al. Rethink the expansion of access and benefit sharing. Science 367, 1200-1202 (2020).

9. Karger, E., du Plessis, P., Meyer, H. Digital Sequence Information on Genetic Resources (DSI) - An introductory guide for African policymakers and stakeholders (German Society for International Cooperation, GIZ, 2019).

10. Friso, F. et al. Implementation of Nagoya Protocol on access and benefit-sharing in Peru: Implications for researchers. J. Ethnopharmacol. 259, 112885 (2020).

11. Alves, R. J. V. et al. Brazilian legislation on genetic heritage harms Biodiversity Convention goals and threatens basic biology research and education. An. Acad. Bras. Cienc. 90, 1279-1284 (2018).

12. Williams, C. et al. Conservation Policy: Helping or hindering science to unlock properties of plants and fungi. Plants People Planet 2, 535-545 (2020).

13. dos S. Ribeiro, C., Koopmans, M. P., Haringhuizen, G. B. Threats to timely sharing of pathogen sequence data - The Nagoya Protocol may impose costs and delays. Science 362, 404-406 (2018).

Acknowledgement: Amber Scholz, Balakrishna Pisupati, Manuela da Silva, Frank Michiels, and Felicity Keiper are gratefully acknowledged for stimulating discussions and valuable suggestions. 